\title{
Experimental Validation of a Radar-based Structural Health Monitoring System
}

\author{
Alexander C. Amies, Christopher G. Pretty, Geoffrey W. Rodgers, and J. Geoffrey Chase
}

\begin{abstract}
This paper presents a new structural health monitoring (SHM) method using frequency-modulated continuous wave (FMCW) radar. The method was developed to circumvent issues with SHM methods' need for displacement measurements, which can be difficult to obtain robustly through integrated accelerations, or through other displacement measurement methods. Instead, interstorey drift ratios were estimated through the direct measurement of interstorey displacement using FMCW radar. Simulation of this method using historical structural response data verified suitably accurate displacement measurements could be obtained using FMCW radar, and prompted the construction of a prototype system. Experimental validation of this prototype was carried out on a shake table. The precision of the system in terms of mean interstorey drift ratio was found to be $1.09 \times 10^{-3}$. These results are encouraging for the future deployment of this SHM approach.
\end{abstract}

Index Terms - radar applications, intelligent structures, simulation.

\section{INTRODUCTION}

$\mathbf{R}$ ADAR is an electromagnetic wave-based technique used to detect the presence of objects and their distance from a transmitting and receiving unit. This unit transmits radio waves of known frequencies and compares these waves to received echoes to determine the distance to nearby reflective objects. There are numerous methods used to determine the distance to reflectors, including time of flight of the signal, and frequency analysis of a frequency modulated radar signal. This paper presents a method employing the latter technique to provide direct displacement measurements for SHM.

SHM in the context of parametric methods applied to multistorey structures requires the ability to measure or estimate the distance a structure displaces from its resting position during perturbation by external forces, including seismic and wind loading. Based on a network of sensors, estimates can be made in real-time about the likelihood of structural damage having occurred, without the need to visibly inspect and test structural elements [1], [2].

There are numerous metrics used to identify likelihood of structural damage, and among these is the interstorey drift ratio (IDR). This metric measures how far one level of a multistorey structure is displaced from its resting position relative to the storey below it, and the distance separating the two storeys. Typical design guidelines use 0.02 IDR as a metric for nonlinear damage [3], [4].

A. C. Amies, C. G. Pretty, G. W. Rodgers, and J. G. Chase were with the Mechanical Engineering Department, University of Canterbury, Christchurch, New Zealand.
Modern methods of measuring displacement typically utilise accelerometers, and then doubly-integrate to obtain displacement estimates. This method is susceptible to integral drift errors [5], resulting in damaging motion potentially going undetected if a structure resettles in a different position after a seismic event. The ability to measure structural displacement avoids the need for baseline correction [6], [7]. This research therefore a non-contact method of measuring structural displacement directly.

There are currently numerous existing types of SHM sensors. These sensors include impedance-measuring devices [8], accelerometers [9], [10], and sensors which measure displacement directly, such as non-contact line scanners [11], [12], [13] and directly-connected linear variable differential transformer (LVDT) methods. The former requires direct line of sight (LOS) between both ends of the measured distance, and the latter requires space in the structure for the system to be installed and can be damaged. To incorporate these methods in a structure, allowances would thus need to be made for the placement of these sensors. This requirement makes such designs less robust and thus difficult to retrofit to existing buildings. A non-line of sight (NLOS), non-contact method was desired, and radar was identified as a suitable technology for this purpose. Doppler radar methods have been shown to be suitable in the monitoring of wind turbine structures [14], [15].

In order to obtain displacement measurements, FMCW radar can be used. This method transmits a signal with time-varying frequency, and compares the returned echo of the signal with the transmitted signal at the same instant by multiplying (or heterodyning) the two signals to obtain a beat signal. The fundamental frequency of this mixed signal is proportional to the signal time-of-flight. From this value, a distance can be obtained. The parameters of the FMCW system, including bandwidth, centre frequency, modulation sweep time, and signal amplification, determine how successful the system is at detecting small perturbations in distance and at detecting distant objects.

This paper explores how such a system would be implemented, and its suitability for SHM. The particular application of this form of radar in an SHM context is a novel approach to monitoring structures undergoing seismic excitation. The small perturbations which can be measured using FMCW radar are shown to allow for precise determinations to be made regarding post-event structural health. 


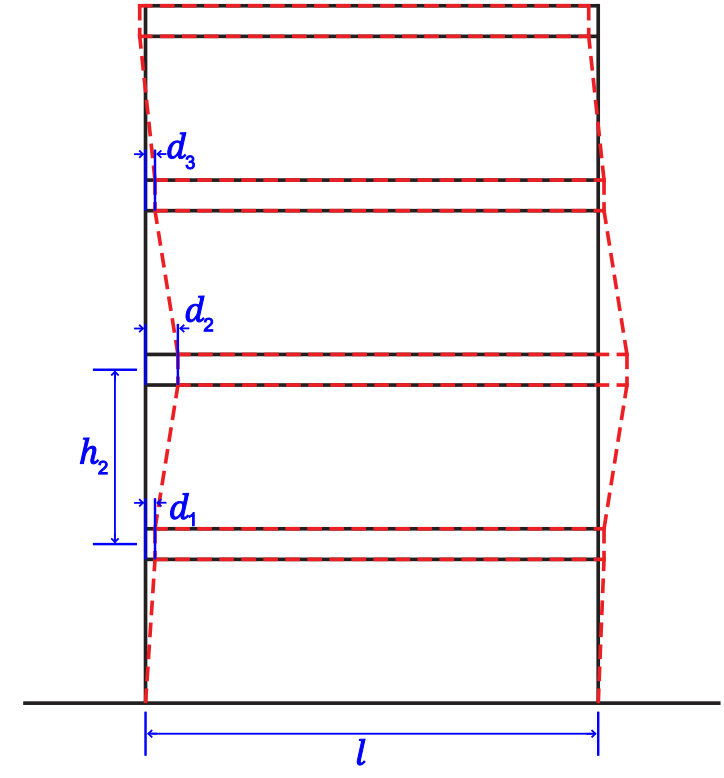

Fig. 1. A building before (black) and during (red) a seismic event. The quantities used to calculate the IDR are indicated in blue.

\section{THEORY}

\section{A. Structural Health Monitoring Requirements}

Literature detailing the requirements of SHM systems points to SHM being a problem of statistical analysis [16]. The identification of particular features in data obtained from structural monitoring provides a likelihood of weakening or damage in the relevant structural members. Typically, structural data is obtained from sensors measuring numerous metrics of structural deformation while undergoing loading. The sources of this data include accelerometers to measure the acceleration of different planar surfaces of a structure, strain gauges (including fiber Bragg grating (FBG) devices [17], [18], [9], [10]) to identify the strain specific structural members have undergone, and LVDT sensors and other equivalent linear tools such as laser line scanners [12] to measure the displacement of specific elements. Features of the time-series data captured by each of these sources can identify damage.

One common metric used to identify damage in a multilevel structure is the IDR. This metric expresses the amount a level is displaced from its resting (central) position relative to the distance of separation between the two levels. The instantaneous IDR of the $i^{\text {th }}$ floor, $\delta_{i}$, is defined:

$$
\delta_{i}=\frac{\left(d_{i}-d_{i-1}\right)}{h_{i}}
$$

where $d_{i}$ is the distance from the rest position the floor has travelled, and $h_{i}$ is the height of the $i^{\text {th }}$ floor above the floor below [19]. A diagram showing the parameters of this metric in a hypothetical 2D structure is presented in Figure 1.

IDRs are particularly useful in the determination of damage when applied to parametric models of damage in multi-storey structures, such as the Bouc-Wen model [20], [21]. This model was simplified [22] into the form:

$$
\boldsymbol{M} \cdot \ddot{v}+\boldsymbol{C} \cdot \dot{v}+\boldsymbol{K} \cdot v=-\boldsymbol{M} \cdot \mathbf{1} \ddot{x}_{g}
$$

Here, $\boldsymbol{M}, \boldsymbol{C}$, and $\boldsymbol{K}$ are the mass, damping and stiffness parameters of the structure, $\ddot{x}_{g}$ is the ground acceleration applied to the structure, $\mathbf{1}$ is a column vector of ones with length equal to the number of floors in the structure, and $v, \dot{v}$, and $\ddot{v}$ are the structure's displacement, velocity, and acceleration respectively. Damage resulting from external loading causes a change in structural parameters, in particular natural frequency and stiffness. These changes in behaviour require a remodeled structure with the form:

$$
\boldsymbol{M} \cdot \ddot{v}+\boldsymbol{C} \cdot \dot{v}+(\boldsymbol{K}+\Delta \boldsymbol{K}) \cdot v=-\boldsymbol{M} \cdot \mathbf{1} \ddot{x}_{g}
$$

The additional parameter $\Delta \boldsymbol{K}$ contains the information relating to structural parameter changes and may be timevariant. The goal of SHM systems is to provide the $v, \dot{v}$, and $\ddot{v}$ data such that, with knowledge of ground motion and initial structural parameters, the structural changes post-event can be identified. Hence, understanding of IDRs, related to the $v$ term in Equation (3), is required for parametric SHM methods.

It has been stated that IDRs of $\left|\delta_{i}\right| \geq 0.005$ can be indicative of at least light damage to structural members in a heuristic motion-damage relationship, with $\left|\delta_{i}\right| \geq 0.009$ indicating moderate damage and $\left|\delta_{i}\right| \geq 0.015$ indicating severe damage [23], [24], with $\left|\delta_{i}\right|=0.02$ being a typical design level. However, testing of a variety of specimens has indicated that cracking occurred in members at a range of IDRs (specifically $0.0019 \leq\left|\delta_{i}\right| \leq 0.008$ ) [23]. From this result, the need for probabilistic fragility functions, which depend on both empirical cracking data and the precise peak IDR observed to return a likelihood of structural weakening or failure, has arisen [25], [26], [27]. As such, for a new method of drift detection to meet SHM requirements, the method must be able to detect IDRs below values of 0.002 , while reporting peak IDRs as precisely as possible.

Some other methods of automated damage assessment rely on wavelet transforms to identify probable damaging motion [23], [28], [29], [30]. This involves the transformation of the time-series structural displacement data onto a basis of wavelets known to characterise damage with varying levels of probability. For use with these methods of damage identification and for buildings with responses in the $0.2 \mathrm{~Hz}$ to $20 \mathrm{~Hz}$ range, the proposed SHM method should be able to record measurements at a rate in the region of $50 \mathrm{~Hz}$ to $100 \mathrm{~Hz}$ [31].

\section{B. Obtaining Structural Displacement}

For IDRs to be calculated, the displacement of two consecutive storeys must be known. This value can be obtained by comparing the distance diagonally between the two storeys, defined here as the interstorey diagonal distance (IDD), and is depicted in Figure 2. By observing how the IDD changes during a seismic event, the IDR can be calculated.

1) Radio Wave Detection: Radar is a commonly-used method of distance measurement using radio frequency (RF) waves. This method involves the observation of reflected signals' frequencies relative to the transmitted frequency. Some 


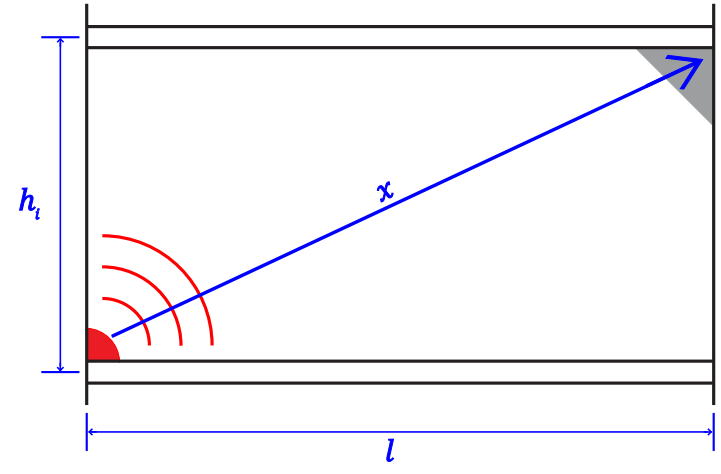

Fig. 2. An example of a hypothetical two-dimensional structure with a radar transceiver placed in the lower left corner and a reflector in the upper right corner. The interstorey diagonal distance, $x$, is represented in blue.

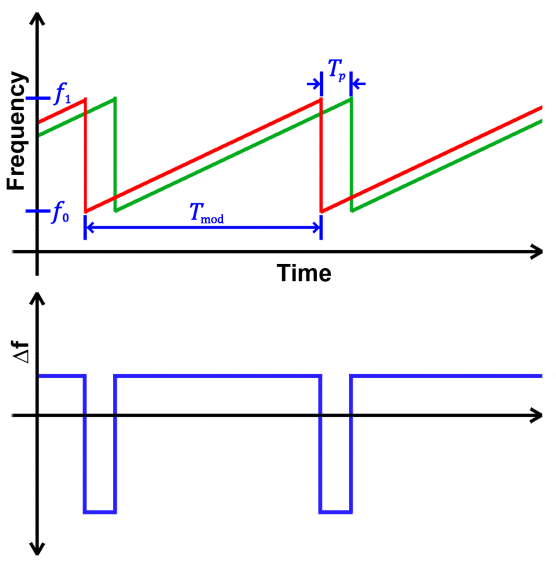

Fig. 3. An example of how frequency-modulated continuous wave signals change in frequency over time. The transmitted signal is shown in red, and the reflected and received signal is shown in green, indicating the delay between the two signals. The difference in frequency between the two signals is shown in blue, indicating that the signals have mostly a constant frequency difference, where the frequency difference is proportional to the time it takes for the wave to return.

methods use Doppler shift of reflected signals to identify moving targets, which allows the object's velocity to be measured. This style of measurement would not be suitable for obtaining IDR data because the target's velocity is returned, which would need to be integrated.

FMCW radar systems transmit a signal with a frequency, which changes over time. For example, a system can use a triangular modulation, in which the signal's frequency increases linearly from a lower frequency bound $\left(f_{0}\right)$ to an upper frequency bound $\left(f_{1}\right)$. Once the transmitter modulator output reaches the upper bound, the output immediately reverts to the lower bound and continues transmitting with its frequency varying in a sawtooth manner, as shown in Figure 3.

The reflected signal is received some time after it was transmitted. The delay means that at the instant the reflected signal arrives at the receiving antenna, the transmitting antenna is sending a signal with a higher frequency. The frequency difference between the signals is proportional to the delay time, and thus the distance the reflected signal travelled. The transmitted and received signals are defined:

$$
\begin{aligned}
v_{\mathrm{FM}}(t) & =A_{c} \cos \left[\omega_{c}(t)+\frac{A_{b}}{2 t^{2}}\right] \\
v_{\mathrm{FM}}\left(t-T_{p}\right) & =A_{c} \cos \left[\omega_{c}\left(t-T_{p}\right)+\frac{A_{b}}{2}\left(t-T_{p}\right)^{2}\right]
\end{aligned}
$$

where $A_{c}$ is the transmitted signal amplitude, $\omega_{c}(t)$ is the signal frequency, $A_{b}$ is the rate of increase of the frequency sweep, and $T_{p}$ is the time taken for the signal to arrive at the receiver [32]. These signals can be heterodyned using an electronic mixer to produce the beat signal defined:

$$
\begin{aligned}
& v_{\text {out }}(t)=v_{\mathrm{FM}}(t) \times v_{\mathrm{FM}}\left(t-T_{p}\right) \\
& v_{\text {out }}(t)=\frac{A_{c}^{2}}{2}\left[\cos \left(m_{\text {out }} t+A_{b} t^{2}+\phi_{u}\right)+\cos \left(A_{b} T_{p} t-\phi_{u}\right)\right]
\end{aligned}
$$

The phase shift for each cosine term in Equation (4), $\phi_{u}$, is equal to $\left(A_{b} / 2\right) T_{p}^{2}-\omega_{c} T_{p}$. The first cosine term in Equation (4) has a linearly increasing frequency, $m_{\text {out }}=$ $2 \omega_{c}-A_{b} T_{p}$, which is approximately twice as large as the carrier frequency, and can thus be filtered using a low-pass filter. This filtering leaves the second cosine term, which has a frequency defined:

$$
f_{r}=\frac{A_{b} T_{p}}{2 \pi}
$$

Because $f_{r}$ is proportional to $T_{p}$, the time taken for the signal to propagate from the transmitter to the receiver, the frequency of this term can be found and used in the FMCW formula [33]:

$$
x=\frac{c \times T_{\text {mod }}}{2\left(f_{1}-f_{0}\right)} f_{r}
$$

where $c$ is the speed of light and the assumed speed of signal transmission, and $T_{\text {mod }}$ is the period over which the linear frequency sweep occurs.

The theoretical limitation of the distance resolution of FMCW systems depends on the discretisation of the beat signal. The Discrete Fourier Transform (DFT) process has a frequency bin resolution of $\frac{1}{T}$, where $T$ is the duration of the signal collected (in this case, $T=T_{\mathrm{mod}}$ ). If the resolution of $f_{r}$ is $\frac{1}{T_{\bmod }}$, then from Equation (5), it follows that:

$$
\begin{aligned}
\Delta x & =\frac{c \times T_{\mathrm{mod}}}{2\left(f_{1}-f_{0}\right)} \Delta f_{r} \\
& =\frac{c \times T_{\mathrm{mod}}}{2\left(f_{1}-f_{0}\right)} \frac{1}{T_{\mathrm{mod}}} \\
& =\frac{c}{2\left(f_{1}-f_{0}\right)}
\end{aligned}
$$

Equation (6) implies increasing the frequency modulation bandwidth improves the distance resolution of the system. While sensor resolution can thus be improved infinitely with increasing sweep bandwidth, practical sweep generators are nonlinear (i.e. stepped), which limits the effective precision 
capabilities of an FMCW system [32]. The precision-limiting effects of these non-linearities can be mitigated by increasing $T_{\text {mod }}$. However, this approach creates a trade-off between spatial precision and temporal resolution.

There are existing techniques to improve fundamental frequency estimation in spectral analysis of signals [34], [35]. They can be applied to the beat signal to interpolate its frequency spectrum. This process means that instead of simply identifying the peak of the beat signal Fast Fourier Transform (FFT) and using that frequency to calculate $x$, the peak of the interpolation can be used, allowing for better estimation of the radar signal distance. Furthermore, zero-padding the signal and applying the FFT creates a spectrum with more frequency bins, but with an added sinc function component. Using interpolation techniques, this approach can also improve frequency estimation accuracy [36], [37]. These methods are utilised in the experimentation detailed in this paper.

There are existing examples of systems which utilise FMCW radar for displacement detection in structures [38], [39], [40]. These devices are placed off-structure and measure the displacement of specific points of the structure. While this method provides a metric for structural deformation, it does not directly relate to the IDR metric for which design and fragility functions exist. As such, the method proposed in this paper measures IDDs so that they can be related to IDRs so that probabilities of structural damage can be simply estimated. Other research indicates that indoor positioning can be performed using FMCW methods with sufficient accuracy to be implemented as an SHM method [41], [42].

2) Mapping Interstorey Diagonal Drift to Interstorey Drift Ratio: For a hypothetical two-dimensional structure with uniform floor length, $l$, as depicted in Figure 2, the cornerto-corner distance (or IDD) measured by a radar system, $x$, is:

$$
x=\sqrt{l^{2}+h_{i}^{2}}
$$

This formulation assumes that the floor length is constant for all floors in the structure. In situations where different floor dimensions are present, a different formula is required. When the structure is disturbed by an external force, $x$ is altered. Assuming that $l$ and $h_{i}$ remain constant during the event, the disturbed $l^{\prime}$ and $h_{i}^{\prime}$ are defined:

$$
\begin{aligned}
l^{\prime} & =l+\left(d_{i}-d_{i-1}\right) \\
h_{i}^{\prime} & =\sqrt{h_{i}^{2}-\left(d_{i}-d_{i-1}\right)^{2}}
\end{aligned}
$$

Through substitution and rearrangement, the relationship between $x^{\prime}, l$ and $h_{i}$ becomes:

$$
x^{\prime}=\sqrt{l^{2}+2 l\left(\delta_{i} h_{i}\right)+h_{i}^{2}}
$$

Rearranging gives:

$$
\delta_{i}=\frac{{x^{\prime}}^{2}-l^{2}-h_{i}{ }^{2}}{2 l h_{i}}
$$

Equation (8) converts IDDs to IDRs.

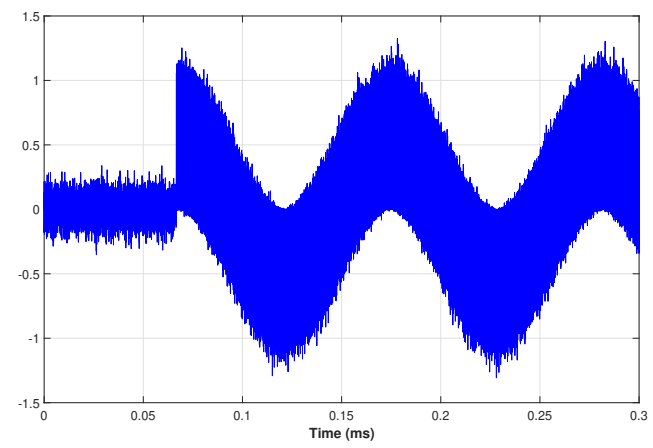

(a) Time domain

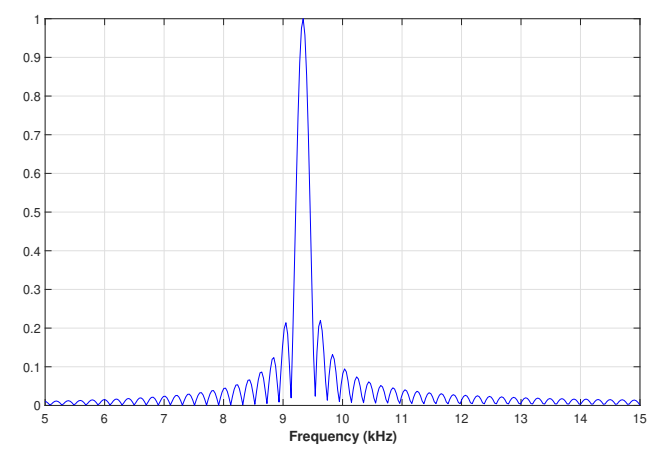

(b) Frequency domain

Fig. 4. Beat signal for a simulated static target $\left(d=10 \mathrm{~m},\left(f_{1}-f_{0}\right)=\right.$ $700 \mathrm{MHz}, T_{\mathrm{mod}}=5 \mathrm{~ms}$, AWGN SNR $\left.=20 \mathrm{~dB}\right)$.

\section{Feasibility and Design Simulation}

Before a radar prototype system was built, an FMCW system was simulated using MATLAB 2016b (The MathWorks, Natick, MA, USA). Simulation enables the testing of a range of frequency sweep bandwidths and modulation timespans to determine the requirements of such a system in an SHM application to measure IDRs $\leq 0.002$. It is thus a feasibility and design analysis.

A single degree of freedom (DOF) target was simulated with a one-dimensional channel, using a previously documented methodology [43]. A static target implemented using this method resulted in the heterodyned response depicted in Figure 4, with a single frequency peak indicating the target's position. Data taken from a structure in the Christchurch Botanic Gardens with period $T=1.0 \mathrm{~s}$ during the 2010 Canterbury, New Zealand Earthquake was used as the tracked target motion. A plot of this data is shown in Figure 5. Because the mean of this data is $0 \mathrm{~m}$, the signal was recentred to a distance of $20 \mathrm{~m}$, and the amplitude was scaled such that the IDDs being simulated corresponded to a peak IDR of 0.015 . These figures were chosen to represent an arbitrary structure undergoing damaging peak motion. Additive white Gaussian noise (AWGN) was inserted into the transmission channel with a signal-to-noise ratio (SNR) of $20 \mathrm{~dB}$ to better simulate realworld channel interference.

As shown in Equation (6), the FMCW resolution is improved with increased sweep bandwidth. With improved spatial resolution, there is an expectation that a radar system's 


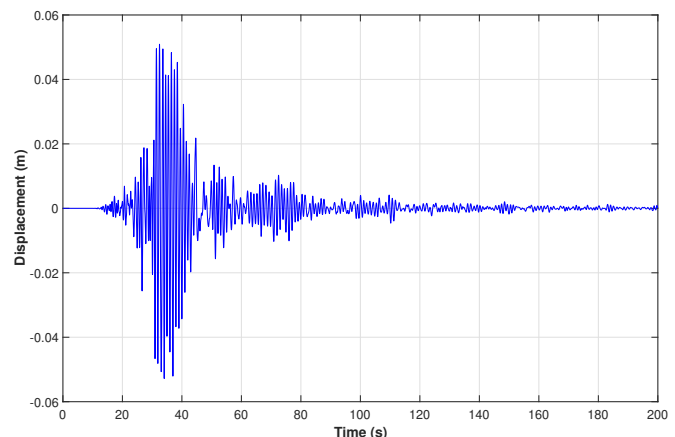

Fig. 5. The data used to simulate a single DOF structure, taken from the 2010 Canterbury, New Zealand earthquake.

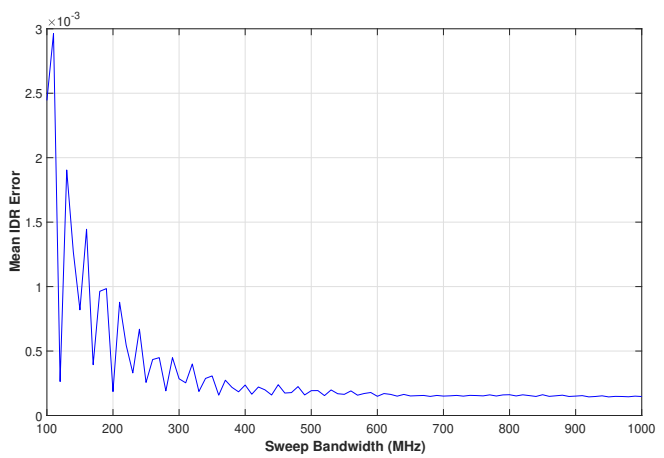

Fig. 6. IDR estimation precision is improved with increased frequency sweep bandwidth. In each case, $T_{\bmod }=5 \mathrm{~ms}$, and $f_{1}=1 \mathrm{GHz}$.

precision is also improved because the frequency bin corresponding to the heterodyned signal's beat frequency is more narrowly-defined. To verify this relationship, simulations across a $5 \mathrm{~s}$ section of the data in Figure 5 were run with the following parameters:

- $f_{0}=9 \mathrm{GHz}$ to $9.9 \mathrm{GHz}$

- $f_{1}=10 \mathrm{GHz}$

- $T_{\text {mod }}=5 \mathrm{~ms}$, therefore data rate $f_{s}=200 \mathrm{~Hz}$

- $\mathrm{AWGN}$ SNR $=20 \mathrm{~dB}$

The results of these simulations are shown in Figure 6. It can be observed that the postulated resolution-precision relationship holds, and for an SHM application, a sweep bandwidth of $120 \mathrm{MHz}$ should be sufficient for the required IDR precision, because the mean IDR error for all sweep bandwidths above this value is smaller than the required detectable IDR of 0.002 .

The level of precision achievable by this method appeared to reach an asymptotic IDR error of $1.50 \times 10^{-4}$ with sweep bandwidths greater than $600 \mathrm{MHz}$. Because of this result, a simulation across the entire data set presented in Figure 5 was run with a bandwidth of $700 \mathrm{MHz}$. In this case, the relative mean distance estimation error was $3.70 \times 10^{-3}$, which corresponded to a mean IDR error of $2.52 \times 10^{-4}$. Notably, the difference between the actual peak IDR and the maximum detected IDR was $1.02 \times 10^{-3}$. These results showed a radarbased SHM system could measure sufficiently small IDRs to detect damaging motion, while being precise enough to

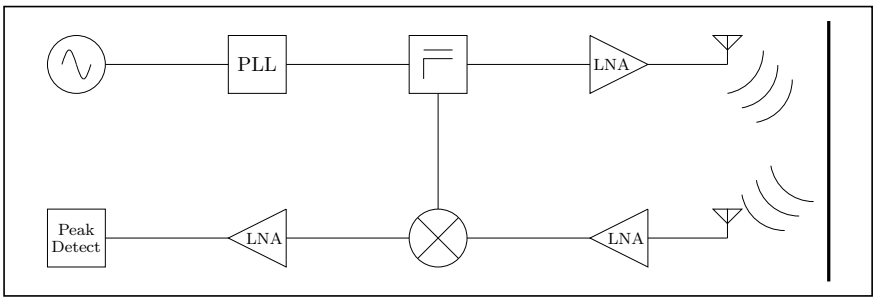

Fig. 7. The functional layout of the FMCW system used to validate this SHM method.

accurately indicate the integrity of a structure. These findings justified the construction and testing of a prototype FMCW device for shake table testing.

\section{IMPLEMENTATION}

The components selected for the prototype were chosen to allow for a large range of sweep bandwidths and modulation times to be tested. Further, discrete modules were used to simplify construction and assembly.

\section{A. Electrical Design}

Figure 7 illustrates the design of the FMCW system created to validate the experimental results. The system was designed with reference to previously instantiated radar setups [44], [45], with components selected to suit the specific SHM application. The requirements of such a system included antennas suited to measuring IDDs. Specifically, antennas were chosen for their relatively narrow beamwidth. Narrow beamwidth enables the antennas to be directed at the reflector, which, due to the nature of damaging but non-destructive structural motion, is expected to have relatively small motion, while limiting the multipath interference expected in this application.

1) Radar Functional Design: The design uses a phaselocked loop (PLL) to modify an oscillator signal to generate the sweep. The PLL parameters are able to be manipulated through software to change the frequency properties of the generated signal to replicate a frequency sweep pattern. An external square wave function generator was used to trigger the ramp start for the distance sampling rate to be varied. The signal output from the PLL was divided, with one output sent to the transmitter antenna, while the other was mixed with the receiver input. The transmitter antenna signal was amplified with a low-noise amplifier (LNA) before being transmitted by a log-periodic antenna, chosen for its directionality combined with its range of frequencies able to be transmitted with relatively constant antenna gain.

The received signal was amplified and mixed with the PLL output, and the intermediate frequency (IF) signal was amplified and collected by a PC-connected analog-to-digital converter (ADC). This signal was then processed with an FFT to find the frequency of the interference signal to obtain the distance transmitted.

2) Radar Components: Components with configurable parameters were selected to determine the limits of operation for such a system in the context of SHM. Specifically, the $T_{\text {mod }}$ and frequency sweep parameters needed to be variable to 
TABLE I

PROTOTYPE FMCW SYSTEM COMPONENTS

\begin{tabular}{lll}
\hline Function & Manufacturer & Component \\
\hline VCO and PLL & Texas Instruments & LMX2492EVM \\
Power Divider & Fairview Microwave & MPR18F-2 \\
Power Amplification & Mini-Circuits & ZVA-183+ \\
Antennas & Aaronia & HyperLOG 60180 \\
Mixer & Hittite Microwave & HMC1048LC3B \\
IF Amplification & Texas Instruments & INA118 \\
ADC & Saleae & Logic Pro 16 \\
Cabling & Generic & RG405 \\
\hline
\end{tabular}

quantify the accuracy and sampling time necessary for SHM. Table I lists the prototype components.

The LMX2492EVM printed circuit board (PCB) contained both a voltage-controlled oscillator and a fractional phaselocked loop. The VCO had a maximum bandwidth of $700 \mathrm{MHz}$ centred around $9.75 \mathrm{GHz}$; this was deemed to be a sufficiently wide bandwidth to test a variety of sweep bandwidths and to achieve the necessary detection accuracy to detect structurally damaging motion. The remaining components were selected due to their near-linear frequency and phase response across this frequency range. The power amplifiers, in addition to their linear response, had a typical gain across the band of $26 \mathrm{~dB}$, which was calculated to be sufficiently large to be used across a testing range of up to $5 \mathrm{~m}$.

\section{B. Reflector Design}

A reflector was designed to act as a target to be tracked by the FMCW radar SHM system. A corner reflector was chosen due to numerous features that made it a more suitable design than a flat sheet reflector. The primary factor was the corner reflector's ability to preserve signal transmission distance regardless of the angle of incidence of the signal. Figure 8 shows the path of two beams to illustrate how this process works. Additionally, the fact the beams exit the reflector in the same, but opposite, direction they entered maximises its reflectivity for systems in which there is little spatial separation between transmitter and receiver.

A corner reflector was also deemed to be a suitable reflector in a structural context. To measure IDDs, the transceiver unit should be placed in one corner of a storey, while the reflector should be placed in the opposite corner. Due to the required corner positioning, a reflector which fits this shape would require the least amount of structural modification or allowance to be fitted. As such, this design was implemented for experimental testing. It was constructed using $1.6 \mathrm{~mm}$ aluminium sheet with an edge length of $370 \mathrm{~mm}$, and is depicted in Figure 9.

\section{EXPERIMENTAL VALIDATION}

The reflector designed was fastened to a shake table, which was driven with ground motion data from five locations near the epicentre of the 2011 Christchurch, New Zealand earthquake. These locations include the Christchurch Resthaven (REHS), Christchurch Cathedral College (CCCC), Canterbury Botanical Gardens (CBGS), Papanui High School (PPHS), and

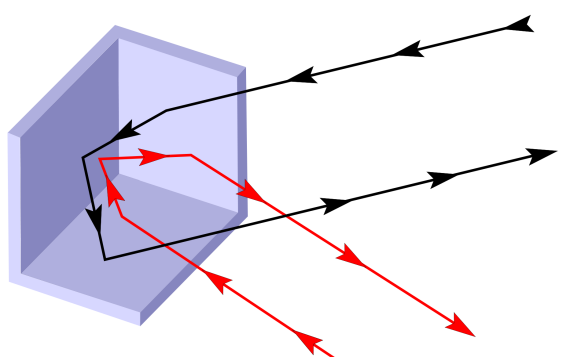

Fig. 8. Corner reflectors reflect incoming beam paths off each of the three internal faces before returning the beam in a direction parallel to the path in which it entered the reflector. Note that regardless of the entry angle, the beam travels the same distance before it exits the reflector.

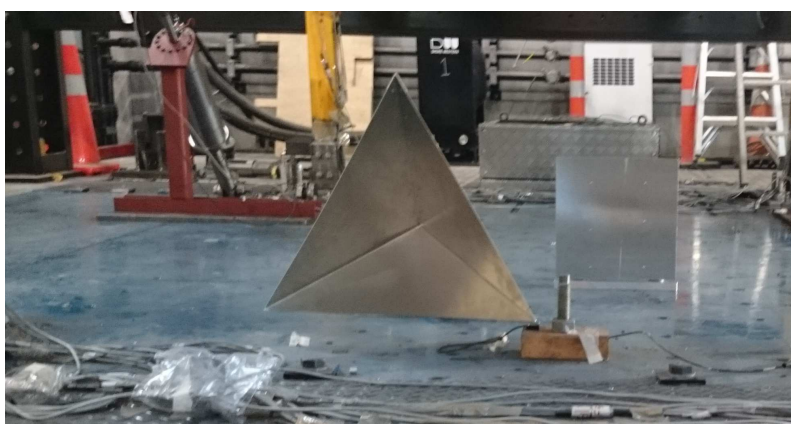

Fig. 9. The corner reflector fixed to a shake table.

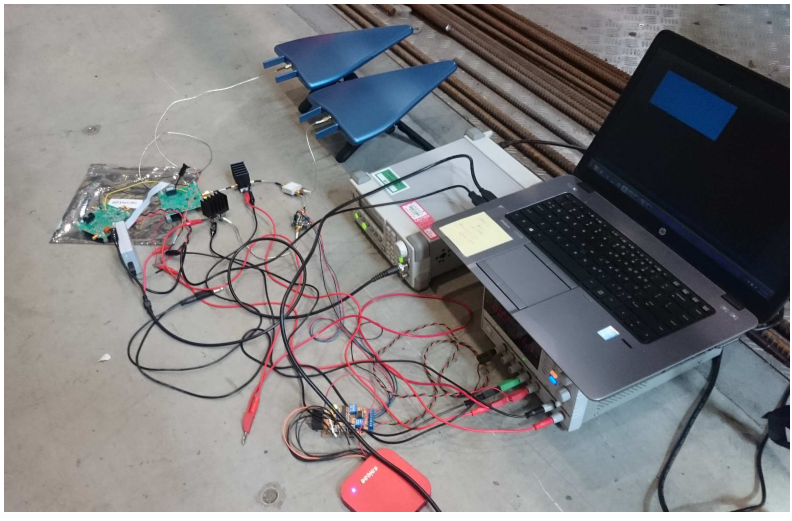

Fig. 10. The prototype FMCW system set up to detect the motion of a shake table. The antennas (blue, top of image) are aimed at the corner reflector shown in Figure 9, and connected to the LMX2492EVM signal generator. The Logic Pro 16 ADC device is shown in red at the bottom of the photo.

Christchurch Hospital (CHHC) strong motion stations [46]. The frequency spectrum of the seismic data, in combination with the amplitude of the shake table $( \pm 0.2 \mathrm{~m})$ made this data suitable motion to validate the ability of the system to detect damaging IDRs in realistic structural responses.

The setup is pictured in Figure 10. The transmitting and receiving antennas were placed as close to each other as possible, and they were both placed around $2 \mathrm{~m}$ from the reflector. The system's $T_{\text {mod }}$ was set to $5 \mathrm{~ms}$ to match the value used in simulation for ease of comparison.

Each set of seismic data was run in turn on the shake table, with an LVDT sensor used to record to reference position for accuracy validation purposes. The beat signal was captured 
TABLE II

EXPERIMENTAL VALIDATION OF THE PROPOSED FMCW SYSTEM

\begin{tabular}{lccc}
\hline & Mean IDD Error & Mean IDR Error & Peak IDR Error \\
\hline REHS & $0.0279 \%$ & $7.65 \times 10^{-4}$ & $8.04 \times 10^{-5}$ \\
CCCC & $0.0336 \%$ & $1.38 \times 10^{-3}$ & $1.68 \times 10^{-3}$ \\
CBGS & $0.0310 \%$ & $1.06 \times 10^{-3}$ & $1.14 \times 10^{-3}$ \\
PPHS & $0.0329 \%$ & $7.79 \times 10^{-4}$ & $2.76 \times 10^{-4}$ \\
CHHC & $0.0353 \%$ & $1.45 \times 10^{-3}$ & $3.66 \times 10^{-3}$ \\
\hline Mean & 0.0321 & $1.09 \times 10^{-3}$ & $1.37 \times 10^{-3}$ \\
\hline
\end{tabular}

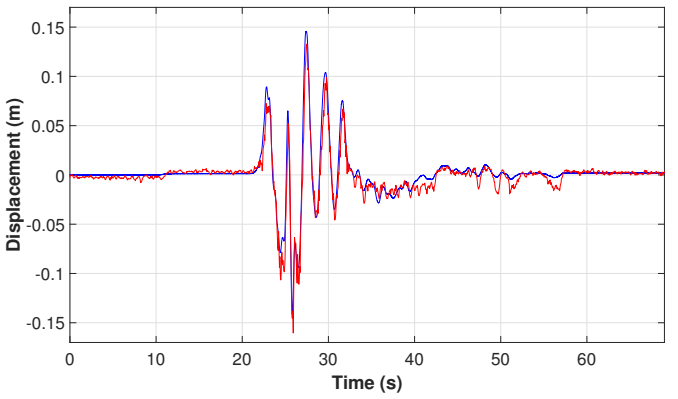

Fig. 11. Seismic motion from REHS run on the shake table. The LVDT data (blue) and radar measurements (using the Peak method, red) are shown.

on the FMCW system, and a simple peak detection method of the spectrum of each sweep was used to obtain the tracked displacement. Because there was no time synchronisation information available, the tracked data and reference data sets were cross-correlated. The delay of the peak correlation was used to align the displacement signals temporally. An example of LVDT and radar data sets plotted together can be seen in Figure 11. The use of this correlation method allowed tracked displacement errors relative to the reference displacement to be calculated.

Table II summarises the results using the same metrics as the simulation. Displacements measured with the FMCW system were treated as IDDs, and then converted to IDRs using Equation (8). Values for $h_{i}$ and $l$ were selected such that the maximum IDR was equal to 0.015 for ease of comparison with the simulated system.

\section{A. Discussion}

While not as precise as was simulated, the FMCW system can detect displacements to a level of accuracy suitable for use in SHM applications. The mean IDR error was $1.09 \times 10^{-3}$, while the error between peak IDRs was $1.37 \times 10^{-3}$. The former value relates comparably to the requirements set for use in SHM in Section II-A, with a mean error almost half the required value.

There were numerous reasons why the theoretical accuracy obtained during simulation was not matched in experimental validation. The main issue is the difficulty of accurately simulating a truly multipath environment. The simulation used additive noise across the entire spectrum. However, in reality, transmitted radar waves reflect in particular patterns and cause groupings at particular beat frequencies. As a result, the 'true' beat frequency, which corresponds to the actual measured

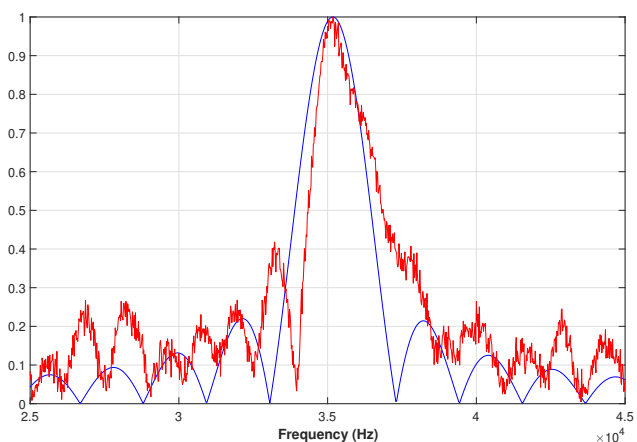

Fig. 12. A comparison of sweep spectra from simulation (blue) and experimental (red) data.

distance using Equation (5), can be hidden due to multipath interference. Figure 12 highlights the difference between these two cases.

Figure 12 also shows the spectral noise of the heterodyned signal captured by the ADC. This noise was not present in the simulated FMCW system. The spectral noise and artifacts present in the plot limit the effectiveness of naïve peak identification as a means of radar distance measurement. From observation of the plot, noise could cause the identified peak to be shifted by as much as $100 \mathrm{~Hz}$ from the 'true' peak. This error corresponds to a relative distance error of $0.3 \%$, a significantly larger error than the mean error found in experimentation. Errors in peak finding could therefore potentially alter the mean IDR error to unacceptable levels. Improved signal processing techniques exist to potentially manage this issue in the future [34], [35].

It was observed that the shape of the spectrum, defined by the relative magnitude of frequencies in the spectrum near to the maximum) remained similar while the reflector was driven. This similarity can be attributed to the multipath nature of the real-world transmission channel. The observed window of the spectrum includes reflections from objects other than the fixed corner reflector, including the shake table itself, and the structure mounted on the table which was simultaneously undergoing testing. These objects were all being driven with motion as the corner reflector, and were constrained in their motion along the same single-DOF axis. The spectral peaks associated with these reflections could potentially be used as additional points of interest to more accurately estimate displacement.

Due to the unconstrained nature of real seismic events, and the multi-DOF response of structures during such events, motion in directions other than the one being measured may significantly alter the channel, and thus the heterodyned spectrum. This lack of constraint could impede the ability of a multiple reflection tracking method to track the target with the required level of precision, due to the change in multipath reflection. However, these differences between shake table and real-world testing should not affect the presented method's ability to identify the reflector. Because this method provides sufficient accuracy for the detection of damaging motion, there is an expectation that an FMCW SHM method should be 
viable, subject to further testing.

The seismic data used to test the FMCW radar system was taken from ground motion data, rather than on-structure data. The use of seismic data meant the measured displacements were of a larger magnitude than some moderately damaging IDRs found in multi-storey structures. This is a limitation of the experiment that makes it a proof-of-concept trial. Further testing of the FMCW radar system with smaller bandwidths is required.

\section{CONCLUSIONS}

The viability of a non-contact an NLOS FMCW system measuring structural displacement directly was explored. The radar-based method presented is non-invasive and thus simpler to retrofit to existing structures. Viability was tested in a twostep process.

Simulation showed FMCW systems with $120 \mathrm{MHz}$ bandwidth could achieve the required level of precision for the detection of damaging structural motion. Additionally, increased sweep bandwidths were shown to improve the precision of displacement measurement as expected.

A prototype system was constructed and tested using a shake table with data from five sites during the 2011 Christchurch, New Zealand earthquake. It was found that a corner reflector target could be tracked with a mean IDR error of $1.09 \times 10^{-3}$, a figure twice as good as required to track identify damaging structural motion. The results of this trial justify the testing of a multi-target system for the purpose of identifying north-south (N-S) and west-east (W-E) IDRs. Further testing of this device in a cluttered environment typical of most structures should also be made before system deployment.

\section{ACKNOWLEDGMENT}

This research was supported by the University of Canterbury College of Engineering Fees Scholarship and a Mechanical Engineering Department Doctoral Scholarship. The authors thank the University of Auckland for use of their shake table to carry out the experimental work detailed in this paper.

\section{REFERENCES}

[1] S. W. Doebling, C. R. Farrar, M. B. Prime, and D. W. Shevitz, "Damage identification and health monitoring of structural and mechanical systems from changes in their vibration characteristics: a literature review," Los Alamos National Lab., NM (United States), Tech. Rep., 1996.

[2] C. R. Farrar and K. Worden, "An introduction to structural health monitoring," Philosophical Transactions of the Royal Society of London A: Mathematical, Physical and Engineering Sciences, vol. 365, no. 1851, pp. 303-315, 2007.

[3] S.-E. Kim and W.-F. Chen, "Practical advanced analysis for unbraced steel frame design," Journal of Structural Engineering, vol. 122, no. 11, pp. $1259-1265,1996$.

[4] B. Ellingwood, "Serviceability guidelines for steel structures," American Institute of Steel Construction Engineering Journal, vol. 26, no. 1, pp. 1-8, 1989.

[5] S. Thenozhi, W. Yu, and R. Garrido, "A novel numerical integrator for structural health monitoring," in 5th International Symposium on Resilient Control Systems (ISRCS). Salt Lake City, UT: IEEE, 2012, pp. 92-97.

[6] D. M. Boore, C. D. Stephens, and W. B. Joyner, "Comments on baseline correction of digital strong-motion data: Examples from the 1999 Hector Mine, California, earthquake," Bulletin of the Seismological Society of America, vol. 92, no. 4, pp. 1543-1560, 2002.
[7] H.-C. Chiu, "Stable baseline correction of digital strong-motion data," Bulletin of the Seismological Society of America, vol. 87, no. 4, pp. 932-944, 1997.

[8] H. Hoshyarmanesh, A. Abbasi, P. Moein, M. Ghodsi, and K. Zareinia, "Design and implementation of an accurate, portable, and timeefficient impedance-based transceiver for structural health monitoring," IEEE/ASME Transactions on Mechatronics, vol. 22, no. 6, pp. 28092814, 2017.

[9] H.-N. Li, D.-S. Li, and G.-B. Song, "Recent applications of fiber optic sensors to health monitoring in civil engineering," Engineering Structures, vol. 26, no. 11, pp. 1647-1657, 2004.

[10] P. Moyo, J. M. W. Brownjohn, R. Suresh, and S. C. Tjin, "Development of fiber Bragg grating sensors for monitoring civil infrastructure," Engineering Structures, vol. 27, no. 12, pp. 1828-1834, 2005.

[11] M. Nayyerloo, "Real-time structural health monitoring of nonlinear hysteretic structures," Ph.D. thesis, Univ. Canterbury, 2011.

[12] M. Nayyerloo, J. G. Chase, A. Millane, C. Muller, A. Malherbe, X. Q. Chen, and G. A. MacRae, "Seismic structural displacement measurement using a line-scan camera: camera-pattern calibration and experimental validation," Journal of Civil Structural Health Monitoring, vol. 1, no. 3, pp. 113-124, 2011

[13] H. Jeon, W. Myeong, J. U. Shin, J. W. Park, H. J. Jung, and H. Myung, "Experimental validation of visually servoed paired structured light system (ViSP) for structural displacement monitoring," IEEE/ASME Transactions on Mechatronics, vol. 19, no. 5, pp. 1603-1611, 2014.

[14] J. Muñoz-Ferreras, Z. Peng, Y. Tang, R. Gómez-García, D. Liang, and C. Li, "Short-range doppler-radar signatures from industrial wind turbines: Theory, simulations, and measurements," IEEE Transactions on Instrumentation and Measurement, vol. 65, no. 9, pp. 2108-2119, Sep. 2016.

[15] J. . Muñoz-Ferreras, Z. Peng, Y. Tang, R. Gómez-García, D. Liang, and C. Li, "A step forward towards radar sensor networks for structural health monitoring of wind turbines," in 2016 IEEE Radio and Wireless Symposium (RWS), Jan 2016, pp. 23-25.

[16] H. Sohn, C. R. Farrar, F. M. Hemez, D. D. Shunk, D. W. Stinemates, B. R. Nadler, and J. J. Czarnecki, "A review of structural health monitoring literature: 1996-2001," Los Alamos National Laboratory Los Alamos, NM, Tech. Rep. LA-13976-MS, 2004.

[17] M. Majumder, T. K. Gangopadhyay, A. K. Chakraborty, K. Dasgupta and D. K. Bhattacharya, "Fibre Bragg gratings in structural health monitoring - present status and applications," Sensors and Actuators A: Physical, vol. 147, no. 1, pp. 150-164, 2008.

[18] T. H. T. Chan, L. Yu, H. Y. Tam, Y. Q. Ni, S. Y. Liu, W. H. Chung, and L. K. Cheng, "Fiber Bragg grating sensors for structural health monitoring of Tsing Ma bridge: Background and experimental observation," Engineering Structures, vol. 28, no. 5, pp. 648-659, 2006.

[19] E. Miranda, "Approximate seismic lateral deformation demands in multistory buildings," Journal of Structural Engineering, vol. 125, no. 4, pp. 417-425, 1999.

[20] R. Bouc, "Forced vibration of mechanical systems with hysteresis," 1967.

[21] Y.-K. Wen, "Method for random vibration of hysteretic systems," Journal of Engineering Mechanics, vol. 102, no. 2, pp. 249-263, 1976.

[22] J. G. Chase, K. L. Hwang, L. R. Barroso, and J. B. Mander, "A simple LMSbased approach to the structural health monitoring benchmark problem," Earthquake Engineering \& Structural Dynamics, vol. 34, no. 6, pp. 575-594, 2004.

[23] F. Naeim, S. Hagie, and A. Alimoradi, "Automated post-earthquake damage assessment and safety evaluation of instrumented buildings," John A. Martin \& Associates, Inc. Research \& Development Department, JAMA Report, 2005

[24] A. Ghobarah, "On drift limits associated with different damage levels," in Performance-Based Seismic Design Concepts and Implementation: Proceedings of the International Workshop, Bled, Slovenia, vol. 28, 2004, pp. 321-332.

[25] C. M. Ramirez, D. G. Lignos, E. Miranda, and D. Kolios, "Fragility functions for pre-Northridge welded steel moment-resisting beam-tocolumn connections," Engineering Structures, vol. 45, pp. 574-584, 2012.

[26] J. W. Baker, "Efficient analytical fragility function fitting using dynamic structural analysis," Earthquake Spectra, vol. 31, no. 1, pp. 579-599, 2015.

[27] E. Miranda, "Use of probability-based measures for automated damage assessment," The Structural Design of Tall and Special Buildings, vol. 15 , no. 1 , pp. 35-50, 2006.

[28] A. Al-Khalidy, M. Noori, Z. Hou, R. Carmona, S. Yamamoto, A. Masuda, and A. Sone, "Health monitoring systems of linear structures 
using wavelet analysis," in International Workshop on Structural Health Monitoring, F.-K. Chang, Ed. Stanford, CA: Technomic, 1997, pp. 164-178.

[29] Z. Sun and C. C. Chang, "Structural damage assessment based on wavelet packet transform," Journal of Structural Engineering, vol. 128, no. 10 , pp. $1354-1361,2002$.

[30] Z. Hou, M. Noori, and R. S. Amand, "Wavelet-based approach for structural damage detection," Journal of Engineering Mechanics, vol. 126 , no. 7, pp. 677-683, 2000.

[31] G.-D. Zhou and T.-H. Yi, "Recent developments on wireless sensor networks technology for bridge health monitoring," Mathematical Problems in Engineering, vol. 2013, p. 33, 2013.

[32] G. M. Brooker, "Understanding millimetre wave FMCW radars," in 1st International Conference on Sensing Technology. Palmerston North, New Zealand: IEEE, 2005, pp. 152-157.

[33] P. Deacon, R. Hunt, D. Koenigsknecht, C. Leonard, and C. Oakley, "Frequency modulated continuous wave (FMCW) radar," 2011.

[34] V. K. Jain, W. L. Collins, and D. C. Davis, "High-accuracy analog measurements via interpolated FFT," IEEE Transactions on Instrumentation and Measurement, vol. 28, no. 2, pp. 113-122, 1979.

[35] F. Zhang, Z. Geng, and W. Yuan, "The algorithm of interpolating windowed FFT for harmonic analysis of electric power system," IEEE Transactions on Power Delivery, vol. 16, no. 2, pp. 160-164, 2001.

[36] M. Abe and J. O. Smith III, "Design criteria for the quadratically interpolated FFT method (i): Bias due to interpolation," no. STAN-M114, 2004.

[37] S. M. Spangenberg, I. Scott, S. McLaughlin, G. J. R. Povey, D. G. M. Cruickshank, and P. M. Grant, "An FFT-based approach for fast acquisition in spread spectrum communication systems," Wireless Personal Communications, vol. 13, no. 1, pp. 27-55, 2000.

[38] J. A. Rice, C. Li, C. Gu, and J. C. Hernandez, "A wireless multifunctional radar-based displacement sensor for structural health monitoring," in Sensors and Smart Structures Technologies for Civil, Mechanical, and Aerospace Systems, vol. 7981. San Diego, CA: SPIE, 2011.

[39] C. Gentile, "Radar-based measurement of deflections on bridges and large structures: advantages, limitations and possible applications," in IV ECCOMAS Thematic Conference on Smart Structures and Materials, Porto, Portugal, 2009.

[40] L. Mayer, B. S. Yanev, L. D. Olson, and A. W. Smyth, "Monitoring of the Manhattan Bridge for vertical and torsional performance with GPS and interferometric radar systems," in Transportation Research Board 89th Annual Meeting. Washington, DC, USA: Transport Research Board, 2010.

[41] R. Gierlich, J. Huttner, A. Dabek, and M. Huemer, "Performance analysis of FMCW synchronization techniques for indoor radiolocation," in 2007 European Conference on Wireless Technologies. Munich, Germany: IEEE, 2007, pp. 24-27.

[42] G. Wang, C. Gu, T. Inoue, and C. Li, "Hybrid FMCW-interferometry radar system in the $5.8 \mathrm{GHz}$ ISM band for indoor precise position and motion detection," in IEEE MTT-S International Microwave Symposium Digest. Seattle, WA: IEEE, 2013, pp. 1-4.

[43] A. C. Amies, C. G. Pretty, G. W. Rodgers, and J. G. Chase, "Development of a non-contact, non-line of sight displacement sensor for structural health monitoring," in 2016 New Zealand Society for Earthquake Engineering Conference (NZSEE), Christchurch, New Zealand, 2016.

[44] C. Li, W. Chen, G. Liu, R. Yan, H. Xu, and Y. Qi, "A noncontact FMCW radar sensor for displacement measurement in structural health monitoring," Sensors (Basel, Switzerland), vol. 15, no. 4, pp. 7412-7433, 2015.

[45] S. Scheiblhofer, M. Treml, S. Schuster, R. Feger, and A. Stelzer, "A versatile FMCW radar system simulator for millimeter-wave applications," in Radar Conference, 2008. EuRAD 2008. European, 2008, pp. 447-450.

[46] L. M. Wotherspoon, R. Orense, B. A. Bradley, B. R. Cox, C. Wood, and R. A. Green, "Geotechnical characterization of christchurch strong motion stations," Earthquake Commission (EQC), Tech. Rep., 2013.

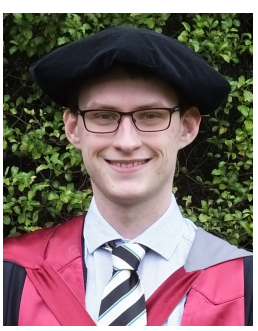

Alexander Amies Dr Alexander Amies is an electrical engineer and data scientist from Christchurch, New Zealand. He completed a $\mathrm{BE}$ (Hons) at the University of Canterbury in 2013, and a PhD at the same institution in 2018. His thesis was centred on a radar-based method of structural health monitoring. His research interests include signal processing, machine learning, sports analytics, and geocomputation. $\mathrm{He}$ is currently employed as a data scientist at Manaaki Whenua - Landcare Research in Lincoln, New Zealand.

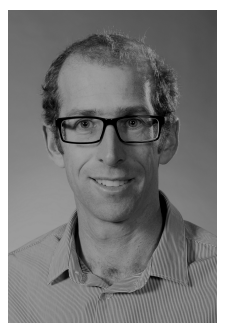

Christopher Pretty Dr Christopher Pretty is currently a senior lecturer in the Department of Mechanical Engineering at the University of Canterbury, NZ. He received his Masters Degree in Mechanical Engineering at the University of Canterbury in 2002, before working in industry for 6 years. Dr Pretty returned to the University of Canterbury and completed a $\mathrm{PhD}$ in Bioengineering in 2012. Following his $\mathrm{PhD}$, he moved to Belgium to undertake a postdoctoral fellowship at the University of Liege in the GIGA Cardiovascular Sciences research group.

Dr Pretty is actively engaged in robotics research for both autonomous systems and assistive robotics. His research has a particular focus on modelling, sensing, and high-accuracy localisation and mapping in outdoor environments. Applications of his work are structural health monitoring, topographic survey, precision agriculture, as well as hybrid exoskeletons for stroke rehabilitation.

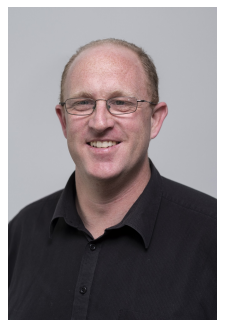

Geoffrey Rodgers Dr Geoffrey Rodgers is a Professor in the Department of Mechanical Engineering at the University of Canterbury, NZ. He received his Bachelors Degree in Mechanical Engineering at the University of Canterbury in 2005, and his $\mathrm{PhD}$ in Mechanical Engineering in 2009, where his thesis was focussed on energy dissipation for earthquake engineering applications. Dr Rodgers then worked as a postdoctoral fellow in biomedical engineering at the University of Otago Christchurch from 2010 to 2012. Dr Rodgers then returned to the University of Canterbury as an academic staff member in late 2012. Dr Rodgers primary research interests are on earthquake engineering and biomedical engineering. His research has a particular focus on energy dissipation/damping devices, instrumentation, and structural health monitoring.

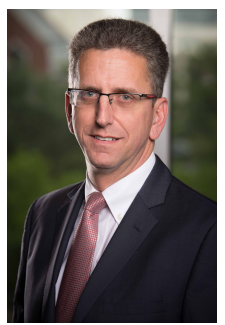

Geoffrey Chase J. Geoffrey Chase received the B.S. in Mechanical Engineering from Case Western Reserve University in 1986, and the M.S. in Mechanical Engineering and the Ph.D. degree in Civil Engineering from Stanford University in 1991 and 1996, respectively. He is currently the Inaugural University of Canterbury Distinguished Professor in the Department of Mechanical Engineering and the Center for Bio-Engineering, University of Canterbury, Christchurch, New Zealand, and an Honorary Clinical Professor at the University of Otago School of Medicine. His current research interests include novel model-based control systems and their implementation in the broad areas of structural engineering, structural health monitoring, and biomedical/clinical engineering. 